\title{
Oxidative arylamination of 1,3-dinitrobenzene and 3-nitropyridine under anaerobic conditions: the dual role of the nitroarenes
}

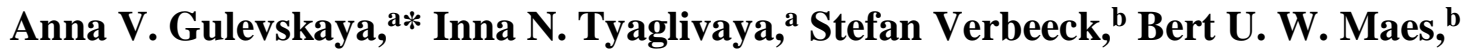 \\ and Anna V. Tkachuk ${ }^{a}$ \\ ${ }^{a}$ Southern Federal University, Department of Chemistry, Zorge str. 7, 344090 Rostov-on-Don, \\ Russian Federation \\ ${ }^{b}$ University of Antwerp, Department of Chemistry, Groenenborgerlaan 171, B-2020 Antwerp, \\ Belgium \\ E-mail: agulevskaya@sfedu.ru
}

\begin{abstract}
1,3-Dinitrobenzene and 3-nitropyridine react with lithium arylamides under anaerobic conditions to produce $N$-aryl-2,4-dinitroanilines and $N$-aryl-5-nitropyridin-2-amines, respectively, in $8-42 \%$ yields.
\end{abstract}

Keywords: Nucleophilic aromatic substitution of hydrogen, oxidative arylamination, 1,3-dinitrobenzene, 3-nitropyridine, $N$-aryl-2,4-dinitroanilines, $N$-aryl-5-nitropyridin-2-amines

\section{Introduction}

The importance of aromatic and heteroaromatic amines is generally known ${ }^{1}$. The most common method for their preparation is nucleophilic aromatic substitution of halide or other nucleofugal groups under conventional ${ }^{2}$ or catalytic conditions ${ }^{3}$. In cases of electron-deficient substrates, such as azines and nitroarenes, the nucleophilic aromatic substitution of hydrogen $\left(\mathrm{S}_{\mathrm{N}}^{\mathrm{H}}\right),{ }^{4}$ including its oxidative ${ }^{5}$ and vicarious ${ }^{6}$ versions, is an attractive alternative to the above methods. This methodology does not require any preliminary introduction of a classical leaving group into an aromatic substrate and need not expensive catalysts or ligands. Mechanistically, the oxidative $\mathrm{S}_{\mathrm{N}}^{\mathrm{H}}$-amination consists of $\sigma^{\mathrm{H}}$-adduct formation and its subsequent oxidative rearomatization (Scheme 1). 


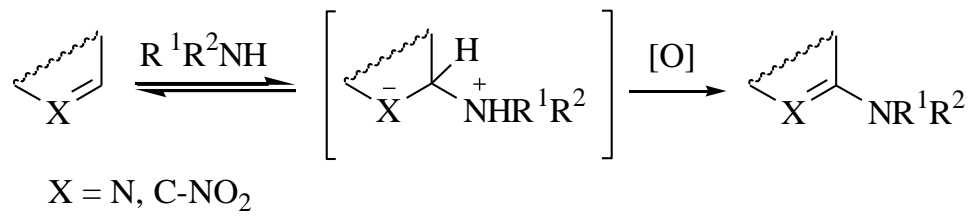

Scheme 1. Oxidative amination of azines and nitroarenes.

In the presence of $\mathrm{KMnO}_{4}$ electron-deficient azines and nitroarenes can be smoothly aminated by potassium amide in a liquid ammonia or liquid ammonia itself. ${ }^{5 a}$ In our previous reports we have shown that an alkylamine/ $\mathrm{AgPy} \mathrm{MnO}_{4}$ system is very efficient for the oxidative alkylamination of fused azines, ${ }^{5 \mathrm{~b}} 3$-nitropyridine ${ }^{5 \mathrm{c}}, 1,3$-dinitrobenzene and nitronaphthalenes ${ }^{5 \mathrm{~d}}$. In the course of this study, we have been interested in the $\mathrm{S}_{\mathrm{N}}^{\mathrm{H}}$-arylamination of aromatic substrates. Unfortunately, the oxidative amination and alkylamination procedure is not suitable for arylamination because of the low nucleophilicity and high sensitivity arylamines towards oxidation. This is why $\mathrm{S}_{\mathrm{N}}^{\mathrm{H}}$-arylamination reactions are still rare. The majority of these are intramolecular and are performed using mild oxidants such as sulfur, chloranil, nitrobenzene. ${ }^{7}$ In some cases, oxidation of $\sigma^{\mathrm{H}}$-complexes with the oxygen in air was observed. ${ }^{8}$ When nitroarenes were used as substrates, the $\mathrm{NO}_{2}$ group itself was often found to be an acceptor of hydride ion. ${ }^{9}$ Similarly, for the amination of azaheterocycles, the ring $\mathrm{C}=\mathrm{N}$ bond can intercept hydride ion. ${ }^{8 \mathrm{~b}}$

To date, only a few intermolecular oxidative arylamination reactions have been reported. 5azacinnoline, ${ }^{10} 3$-X-1,2,4-triazines $\left(\mathrm{X}=\mathrm{SMe}, \mathrm{SBu}^{t}, \mathrm{OMe}\right)^{11}$ and 2-X-3-nitropyridines $(\mathrm{X}=\mathrm{H}$, $\mathrm{Cl})^{12}$ were found to be suitable substrates. In all cases, the reaction was carried out with anilines ${ }^{10}$ or hetarylamines ${ }^{10-12}$ in the presence of a strong base. Thus, to be performed the reaction needs metal arylamide as a nucleophile. Interaction of 5-azacinnoline with arylamines demanded 20 days for completion with crucial access to the air. Arylamination of 1,2,4-triazines proceeded without external oxidant under an inert atmosphere. Obviously, the substrate itself served here as a hydride ion acceptor. 3-Nitropyridines reacted with 2-, 3- or 4-aminopyridines in the presence of LiHMDS (or LDA) in THF without isolation of the reaction mixture from the air oxygen. The use of nitrobenzene as a co-solvent has been shown to improve the yield of the arylamination product. Here we wish to report on the direct arylamination of 3-nitropyridine and 1,3dinitrobenzene by reacting with lithium aryl(hetaryl)amides under anaerobic conditions.

\section{Results and Discussion}

Note, for arylamination of 3-nitropyridines ${ }^{12}, 2-, 3$ - or 4-aminopyridines were solely used as nucleophiles. Checking the feasibility of a similar approach for other aryl- and hetarylamides was our initial goal. We have also yet to answer if this reaction is possible under anaerobic conditions. 
At first, we studied reaction of 3-nitropyridine 1 with 2-aminopyridine under modified experimental procedure (Scheme 2, Table 1). Lithium arylamide was prepared by treating 2aminopyridine $(1.1 \mathrm{mmol})$ with butyl lithium $(1.2 \mathrm{mmol}, 1.6 \mathrm{M}$ solution in hexane $)$ in anhydrous $\mathrm{THF}$ at -78 to $-70{ }^{\circ} \mathrm{C}$ under argon. Solution of 3-nitropyridine $\mathbf{1}$ (1 mmol) in THF was subsequently added. The reaction mixture was stirred for $16-19 \mathrm{~h}$ with gradual warming to room temperature. After extraction and flash column chromatography 5-nitro- $N$-(pyridin-2-yl)pyridin2-amine 2a was obtained in $19 \%$ yields (Table 1, Entry 1). Increased amounts of 2aminopyridine and BuLi led to 2a in higher yields (Table 1, Entries 2-4). The use of 1.6 equiv of arylamine and 1.7 equiv of BuLi was optimal and gave an even better yield of 2a compared with the published ${ }^{11}$ procedure. Upon arylamination of $\mathbf{1}$ with quinolin-2-amine or aniline similar results were observed (Table 1, Entries 10,11,13,14). The corresponding $N$-aryl-5-nitropyridin-2amines $\mathbf{2 b}$ and $\mathbf{2 c}$ were obtained in $34-37 \%$ yields. The procedure was also suitable for amination of 1 with phenylamidine yielding compound 2d (Table 1, Entry 16).

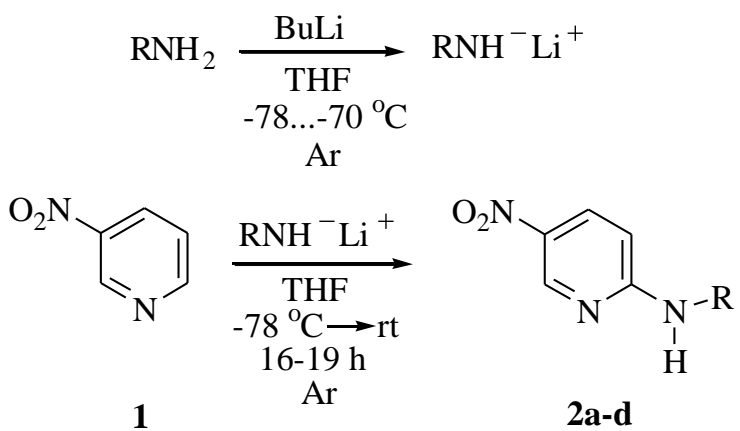

Scheme 2. Synthesis of $N$-aryl-5-nitropyridin-2-amines 2a-d.

The use of nitrobenzene as a co-solvent, as has been shown ${ }^{12}$, improves the yield of the arylamination product due to its ability to accept a hydride ion from $\sigma^{\mathrm{H}}$-adduct and thus, facilitate the rearomatization step. To check this observation, we carried out the reaction of 3 nitropyridine 1 with 2-aminopyridine using nitrobenzene as an additive. Indeed, this additive provided higher yields of 2a (Table 1, Entries 5-7). The use of 1.1 equiv of nitrobenzene was found to be optimal. Addition of nitrobenzene assisted also the reaction of $\mathbf{1}$ with phenylamidine (Table 1, Entry 17). At the same time, its influence on the reactions with other aryl(hetaryl)amines was not essential (Table 1, Entries 12 and 15).

To verify the hypothesis of oxidation with nitrobenzene, we carried out the reaction of 1 with 2-aminopyridine in the presence of 1,2- or 1,3-dinitrobenzene as soon as their oxidative ability is certainly higher. However, 1,2-dinitrobenzene additive gave less yield of 2a (Table 1, Entry 8) than in the reference experiment with nitrobenzene (Table 1, Entry 6). Interestingly, when the reaction of 3-nitropyridine 1 with 2-aminopyridine was carried out in the presence of 1,3-dinitrobenzene $\mathbf{3}$, the arylamino derivatives $\mathbf{2 a}$ and $\mathbf{4 a}$ were obtained in 13 and $28 \%$ yields, 
respectively (Table 1, Entry 9). This indicated that 1,3-dinitrobenzene is more reactive towards amides comparing with 3-nitropyridine.

Table 1. Synthesis of $N$-aryl-5-nitropyridin-2-amines 2a-d

\begin{tabular}{|c|c|c|c|c|c|c|c|}
\hline Entry & Amine & $\begin{array}{l}\text { Equiv } \\
\text { of } \\
\text { amine }\end{array}$ & $\begin{array}{c}\text { Equiv of } \\
\text { BuLi }\end{array}$ & Additive & $\begin{array}{l}\text { Equiv of } \\
\text { additive }\end{array}$ & $\begin{array}{c}\text { Produc } \\
t\end{array}$ & $\begin{array}{l}\text { Yield } \\
(\%)\end{array}$ \\
\hline 1 & & 1.1 & 1.2 & - & - & $2 a$ & 19 \\
\hline 2 & & 1.3 & 1.4 & - & - & $2 a$ & 29 \\
\hline 3 & & 1.6 & 1.7 & - & - & $2 a$ & 41 \\
\hline 4 & & 2.0 & 2.2 & - & - & $2 a$ & 39 \\
\hline 5 & & 1.3 & 1.4 & $\mathrm{PhNO}_{2}$ & 1.1 & $2 a$ & 41 \\
\hline 6 & & 1.6 & 1.7 & $\mathrm{PhNO}_{2}$ & 1.1 & $2 a$ & 52 \\
\hline 7 & & 1.6 & 1.7 & $\mathrm{PhNO}_{2}$ & 2.0 & $2 a$ & 44 \\
\hline 8 & & 1.6 & 1.7 & $\begin{array}{c}1,2- \\
\mathrm{C}_{6} \mathrm{H}_{4}\left(\mathrm{NO}_{2}\right)_{2}\end{array}$ & 1.1 & $2 a$ & 46 \\
\hline 9 & & 1.6 & 1.7 & $\begin{array}{c}1,3- \\
\mathrm{C}_{6} \mathrm{H}_{4}\left(\mathrm{NO}_{2}\right)_{2}\end{array}$ & 1.1 & $\begin{array}{l}2 a \\
4 a\end{array}$ & $\begin{array}{l}13 \\
28\end{array}$ \\
\hline 10 & & 1.3 & 1.4 & - & - & $2 b$ & 27 \\
\hline 11 & & 1.6 & 1.7 & - & - & $2 b$ & 34 \\
\hline 12 & & 1.6 & 1.7 & $\mathrm{PhNO}_{2}$ & 1.1 & $2 b$ & 33 \\
\hline 13 & & 1.3 & 1.4 & - & - & $2 c$ & 38 \\
\hline 14 & & 1.6 & 1.7 & - & - & $2 c$ & 37 \\
\hline 15 & & 1.6 & 1.7 & $\mathrm{PhNO}_{2}$ & 1.1 & $2 c$ & 39 \\
\hline 16 & & 1.6 & 1.7 & - & - & $2 d$ & 22 \\
\hline 17 & & 1.6 & 1.7 & $\mathrm{PhNO}_{2}$ & 1.1 & $2 d$ & 33 \\
\hline
\end{tabular}

As a second part of our work, we examined the arylamination of 1,3-dinitrobenzene $\mathbf{3}$ using the optimized reaction conditions developed for 1 . The results are collected in Scheme 3 and Table 2.<smiles>O=[N+]([O-])c1cccc([N+](=O)[O-])c1</smiles>

3

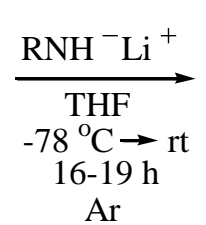

$\mathrm{Ar}$<smiles>[R]Nc1ccc([N+](=O)[O-])cc1[N+](=O)[O-]</smiles>

4a-m

Scheme 3. Synthesis of $N$-aryl-2,4-dinitroanilines 4a-m. 
As shown in Table 2, the nature of the arylamine affects the reaction. Evidently, if the NHacidity of arylamine is high, the corresponding $\mathrm{N}$-anion is formed easier. On the other hand, the greater the $\mathrm{NH}$-acidity of arylamine, the lower the nucleophilicity of the corresponding $\mathrm{N}$-anion. The steric accessibility of the N-nucleophilic center should be also taken into account. The sum of these factors is responsible for yields of the arylamination products 4 . Indeed, as it follows from Table 2 and data on the $\mathrm{NH}$-acidity of arylamines, the highest yields of $\mathbf{4}$ were obtained upon using of 2- and 4-aminopyridines having middle $p K_{a}$ values (Table 2, Entries 1,2). The presence of a substituent in the position ortho to the amino group of arylamine makes difficult the nucleophilic attack and, perhaps, oxidation of the arylamino- $\sigma^{\mathrm{H}}$-complex. That is why compounds $\mathbf{4 g}$ and $\mathbf{4 i}$ were obtained in low yields (Table 2, Entries 7,9). The greater steric hindrance of the peri-amino group may cause reduction in the yield switching from $\mathbf{4 c}$ to $4 \mathbf{d}$ (Table 2, Entries 3,4).

The developed procedure was also suitable for amination of $\mathbf{3}$ with benzamide yielding compound 4m (Table 2, Entry 13).

Table 2. Synthesis of N-aryl-2,4-dinitroanilines 4a-m

\begin{tabular}{|c|c|c|c|c|c|c|c|}
\hline Entry & $\begin{array}{l}\text { Amine } \\
\left(p K_{a}\right)^{\mathrm{a}} \\
\end{array}$ & Product & $\begin{array}{c}\text { Yield } \\
(\%)\end{array}$ & Entry & $\begin{array}{l}\text { Amine } \\
\left(p K_{a}\right)^{\mathrm{a}}\end{array}$ & $\begin{array}{c}\text { Produ } \\
\text { ct }\end{array}$ & $\begin{array}{c}\text { Yield } \\
(\%)\end{array}$ \\
\hline 1 & $(27.7)$ & $4 \mathbf{a}$ & 41 & 8 & & $4 h$ & 28 \\
\hline 2 & & $4 b$ & 42 & 9 & & $4 \mathbf{i}$ & 9 \\
\hline 3 & & $4 c$ & 39 & 10 & & $4 \mathbf{j}$ & 20 \\
\hline 4 & & $4 d$ & 31 & 11 & & $4 k$ & 16 \\
\hline 5 & $(30.6)$ & $4 e$ & 32 & 12 & & $\begin{array}{c}41 \\
5\end{array}$ & $\begin{array}{l}5 \\
8\end{array}$ \\
\hline 6 & (31.0) & $4 f$ & 34 & 13 & & $4 m$ & 12 \\
\hline 7 & & $4 \mathrm{~g}$ & 8 & & & & \\
\hline
\end{tabular}

${ }^{\text {aNH}} \mathrm{NH}$-Acidity in DMSO. ${ }^{13}$ 
It should be mentioned that the reactions of 3-nitropyridine and 1,3-dinitrobenzene with aryl(hetaryl)amides were carried out under anaerobic conditions. Thus, one can conclude that the substrate itself acts here as the oxidant accepting a hydride ion from anionic $\sigma^{\mathrm{H}}$-complex (Scheme 4). That is why the yields of the arylamination products $\mathbf{2}$ and $\mathbf{4}$ did not exceed $42 \%$. In addition, when 2-aminothiazole was used as a nucleophile, the arylamino derivative $4 \mathbf{l}$ was obtained together with 3-nitroaniline 5 (the reduction product of $\mathbf{3}$ ) (Table 2, Entry 12).

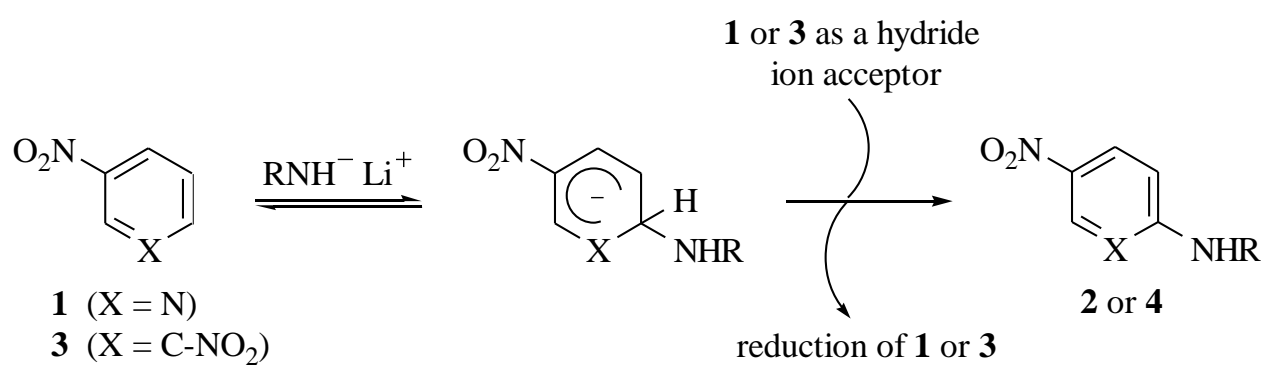

Scheme 4. Mechanism of the compounds 2 and 4 formation.

The structures of compounds 2 and 4 were characterized by UV, IR, ${ }^{1} \mathrm{H}$ NMR, ${ }^{13} \mathrm{C}$ NMR, mass spectroscopy and in two cases (compounds $\mathbf{4 a}$ and $\mathbf{4 k}$ ) single crystal X-ray diffraction analysis. The X-ray data indicate the presence of intramolecular hydrogen bond of the N-H...O type with the length range 1.856-1.898 $\AA$ (Figures $1 \mathrm{~A}$ and 2). Interestingly, the six-membered cycle of $4 \mathbf{a}$ closed by intramolecular hydrogen bond is not planar: the dihedral angle between the $\mathrm{O}-\mathrm{N}-\mathrm{O}$ and $\mathrm{H}-\mathrm{N}-\mathrm{C}$ planes is $\sim 12^{\circ}$ (Figure 1B). Molecule 4k adopts a spiral-like shape: the dihedral angle between the planes of the neighbouring benzene rings is $\sim 33-35^{\circ}$ (Figure 2 ).

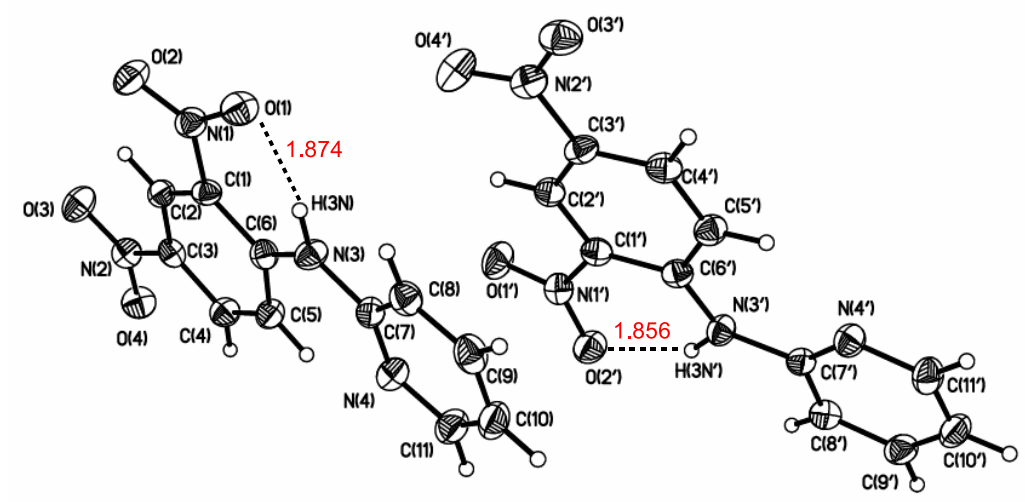

$A$ - ORTEP plots for X-ray crystal structure of $\mathbf{4 a}$ with crystallographic numbering scheme (two independent molecules in the unit cell; the length of intramolecular hydrogen bond is marked in red) 


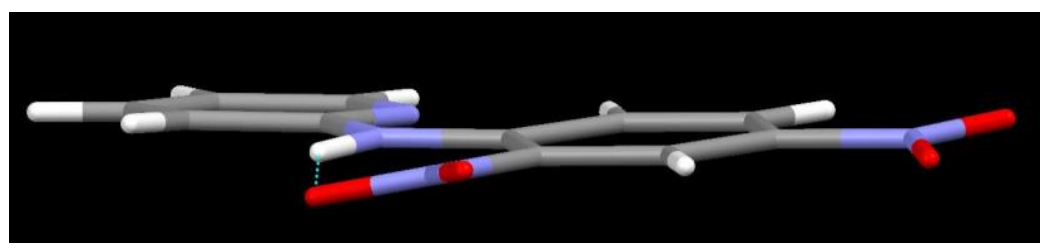

$B$ - view on the molecule $4 \mathbf{a}$, showing that the cycle closed by intramolecular hydrogen bond is not planar $(\mathrm{C}$ - grey, $\mathrm{N}$ - blue, $\mathrm{O}$ - red, $\mathrm{H}$ - white $)$

Figure 1. The X-ray crystal structure of $\mathbf{4 a}$.

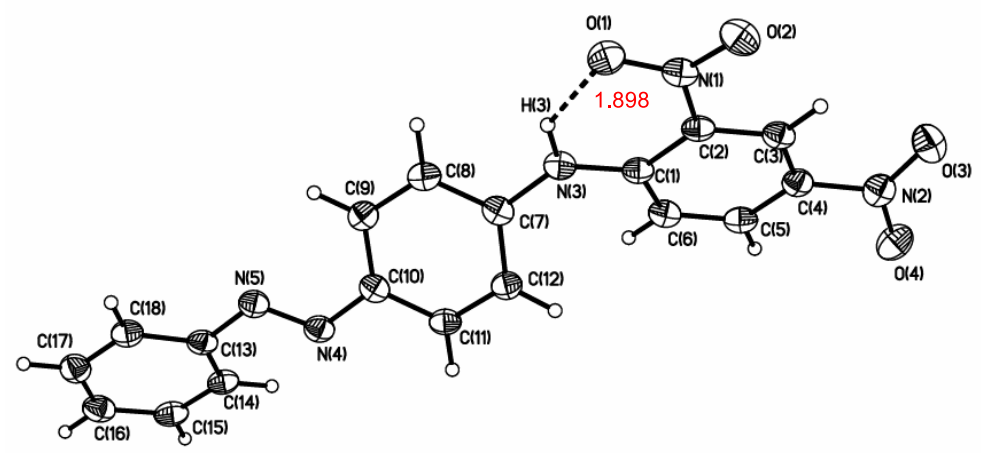

Figure 2. The X-ray crystal structure of $\mathbf{4 k}$ (the length of intramolecular hydrogen bond is marked in red).

\section{Conclusions}

In the present paper we have shown that $N$-aryl-2,4-dinitroanilines and $N$-aryl-5-nitropyridin-2amines may be easily obtained from 1,3-dinitrobenzene and 3-nitropyridine, respectively, through oxidative nucleophilic substitution of hydrogen, using the metal amides of a range of aryl(hetaryl)amines as reactants. The reaction proceeds under anaerobic conditions. The nitroarene plays the dual role in this reaction acting as the substrate and oxidizing agent.

\section{Experimental Section}

General. ${ }^{1} \mathrm{H}$ NMR spectra were obtained on a Bruker DPX-250 (250 MHz) and Bruker Avance $400(400 \mathrm{MHz})$ spectrometers. ${ }^{13} \mathrm{C}$ NMR spectra were recorded on a Bruker DPX-250 (62.9 $\mathrm{MHz})$ and Bruker Avance $400(100 \mathrm{MHz})$ spectrometers. ${ }^{1} \mathrm{H}$ and ${ }^{13} \mathrm{C}$ NMR chemical shifts are in parts per million relative to $\mathrm{Me}_{4} \mathrm{Si}$. Coupling constants are in Hertz. IR spectra were recorded on a Varian Excalibur 3100 FT-IR and Cary 50 Probe spectrometers using Nujol. UV spectra were 
registered on a FSM-1201 spectrophotometer with $\mathrm{CHCl}_{3}$ as a solvent. Mass spectra were measured on a Finnigan MAT INCOS 50 spectrometer. CHN analysis was accomplished by combustion analysis (Dumas and Pregl method). Melting points were determined in glass capillaries using a PTP device and are uncorrected. Column chromatography was performed on silica gel 60 (70-200 mesh; Merck). All commercial reagents (3-nitropyridine, 1,3dinitrobenzene, aryl- and hetarylamines, BuLi, THF) were purchased from Acros and Aldrich and used without additional purification.

Crystallographic data for $\mathbf{4 a}$ and $\mathbf{4 k}$ (atomic coordinates, bond lengths, bond angles and thermal parameters) have been deposited at the Cambridge Crystallographic Data Centre (CCDC) and allocated the deposition number CCDC 815054 (4a) and CCDC815055(4k).

${ }^{1} \mathrm{H}$ and ${ }^{13} \mathrm{C}$ NMR spectra of all $\mathbf{2}$ and $\mathbf{4}$ as well as crystallographic data for $\mathbf{4 a}$ and $\mathbf{4 k}$ are available as supporting information.

\section{General procedure for the synthesis of $N$-aryl-5-nitropyridin-2-amines (2a-d)}

Lithium arylamide was prepared by treating arylamine $(1.6 \mathrm{mmol})$ with butyl lithium $(1.7 \mathrm{mmol}$, $1.6 \mathrm{M}$ solution in hexane) in anhydrous THF $(4 \mathrm{~mL})$ at $-78 \ldots-70{ }^{\circ} \mathrm{C}$ under argon. Solution of 3nitropyridine 1 (124 mg, $1 \mathrm{mmol})$ in THF ( $1 \mathrm{~mL})$ was added dropwise. The resulting mixture was stirred for 16-19 $\mathrm{h}$ with gradual warming to room temperature. The reaction mixture was diluted with water $(5 \mathrm{~mL})$ and extracted with ethyl acetate $(5 \times 10 \mathrm{~mL})$. The organic extract was dried over $\mathrm{MgSO}_{4}$, concentrated and purified by silica gel chromatography using dichloromethane/methanol $(100: 1)$ mixture as the eluent. The bright yellow fraction was collected. Subsequent recrystallization of the crude product from heptane or toluene gave $\mathbf{2}$ in the yield pointed in Table 1.

5-Nitro- $N$-(pyridin-2-yl)pyridin-2-amine (2a). Yellow needles, mp 200-201 ${ }^{\circ} \mathrm{C}$ (heptane), ref. ${ }^{14}$ 196-197.5 ${ }^{\circ} \mathrm{C} .{ }^{1} \mathrm{H} \mathrm{NMR}\left(\mathrm{CDCl}_{3}, 400 \mathrm{MHz}\right): \delta 9.16(\mathrm{dd}, J=2.8,0.6 \mathrm{~Hz}, 1 \mathrm{H}, 6-\mathrm{H}), 8.40(\mathrm{dd}, J=$ 9.3, $2.8 \mathrm{~Hz}, 1 \mathrm{H}, 4-\mathrm{H}), 8.37$ (ddd, $\left.J=5.0,1.9,0.8 \mathrm{~Hz}, 1 \mathrm{H}, 6^{\prime}-\mathrm{H}\right), 7.89$ (d, $\left.J=9.3 \mathrm{~Hz}, 1 \mathrm{H}, 3-\mathrm{H}\right)$, 7.87 (br s, 1H, NH), 7.72 (ddd, $\left.J=8.3,7.3,1.9 \mathrm{~Hz}, 1 \mathrm{H}, 4^{\prime}-\mathrm{H}\right), 7.49$ (dm, $\left.J=8.3 \mathrm{~Hz}, 1 \mathrm{H}, 3^{\prime}-\mathrm{H}\right)$, $7.03\left(\mathrm{ddd}, J=5.0,7.3,0.9 \mathrm{~Hz}, 1 \mathrm{H}, 55^{\prime}-\mathrm{H}\right) .{ }^{13} \mathrm{C}$ NMR $\left(\mathrm{DMSO}_{-}, 100 \mathrm{MHz}\right) \delta 158.4,153.4$, 148.1, 145.8, 138.6, 137.6, 133.6, 118.6, 113.8, 111.0. IR: $3269(\mathrm{~N}-\mathrm{H}), 1496$ and $1331 \mathrm{~cm}^{-1}$ $\left(\mathrm{NO}_{2}\right)$. UV, $\lambda_{\max }(\lg \varepsilon): 290 \mathrm{sh}$ (2.56), 362 (3.31), end absorption up to $443 \mathrm{~nm}$. MS, $m / z$ (I $\geq$ 10\%): 216 (100) [M] $]^{+}, 215$ (77), 170 (23), 169 (39) [M-HNO $]^{+}, 79$ (16), 78 (71), 52 (13), 51 (21). Anal. Calcd for $\mathrm{C}_{10} \mathrm{H}_{8} \mathrm{~N}_{4} \mathrm{O}_{2}$ : C, 55.55; H, 3.73; N, 25.91\%. Found: C, 55.39; H, 4.04; N, $25.72 \%$.

$\boldsymbol{N}$-(5-Nitropyridin-2-yl)quinolin-2-amine (2b). Yellow powder, mp 235-237 ${ }^{\circ} \mathrm{C}$ (toluene). ${ }^{1} \mathrm{H}$ NMR (DMSO-D $6,400 \mathrm{MHz}): \delta 9.16\left(\mathrm{dd}, J=2.8,0.5 \mathrm{~Hz}, 1 \mathrm{H}, 6^{\prime}-\mathrm{H}\right), 8.66\left(\mathrm{~d}, J=9.3 \mathrm{~Hz}, 1 \mathrm{H}, 3^{\prime}-\right.$ H), $8.55\left(\mathrm{dd}, J=9.3,2.8 \mathrm{~Hz}, 1 \mathrm{H}, 4^{\prime}-\mathrm{H}\right), 8.29$ (d, $\left.J=8.9 \mathrm{~Hz}, 1 \mathrm{H}, 4-\mathrm{H}\right), 7.84-7.88$ (m, 3H, 5-H, 8$\mathrm{H}$ and NH), 7.70 (ddd, $J=7.0,8.4,1.5 \mathrm{~Hz}, 1 \mathrm{H}, 6-\mathrm{H}), 7.68$ (d, $J=8.9 \mathrm{~Hz}, 1 \mathrm{H}, 3-\mathrm{H}), 7.46$ (ddd, $J$ $=7.0,8.0,1.1 \mathrm{~Hz}, 1 \mathrm{H}, 7-\mathrm{H}) .{ }^{13} \mathrm{C}$ NMR (DMSO-D, $\left.100 \mathrm{MHz}\right): \delta 158.2,152.6,146.7,145.6$, 138.5, 138.2, 134.0, 130.4, 128.2, 127.4, 125.2, 124.9, 115.0, 111.6. IR: $3356(\mathrm{~N}-\mathrm{H}), 1495$ and $1327 \mathrm{~cm}^{-1}\left(\mathrm{NO}_{2}\right) . \mathrm{UV}, \lambda_{\max }(\lg \varepsilon): 300$ (2.86), 328 (2.97), 370 (3.44), end absorption up to 480 
nm. MS, $m / z$ (I $\geq 10 \%): 266$ (75) $[\mathrm{M}]^{+}, 265$ (63), 220 (24), 219 (41) [M-HNO $]^{+}, 129$ (25), 128 (100), 110 (14), 102 (12), 101 (29), 77 (17), 51 (15). Anal. Calcd for $\mathrm{C}_{14} \mathrm{H}_{10} \mathrm{~N}_{4} \mathrm{O}_{2}$ : C, 63.15; H, $3.79 ; \mathrm{N}, 21.04 \%$. Found: C, 62.87; H, 4.03; N, 21.36\%.

5-Nitro- $N$-phenylpyridin-2-amine (2c). Yellow needles, mp 135-136 ${ }^{\circ} \mathrm{C}$ (heptane), ref..$^{15} 135$ ${ }^{\circ} \mathrm{C} .{ }^{1} \mathrm{H} \mathrm{NMR}\left(\mathrm{CDCl}_{3}, 250 \mathrm{MHz}\right): \delta 9.08(\mathrm{~d}, J=2.7 \mathrm{~Hz}, 1 \mathrm{H}, 6-\mathrm{H}), 8.24(\mathrm{dd}, J=9.3,2.7 \mathrm{~Hz}, 1 \mathrm{H}$, 4-H), 7.34-7.45 (m, 4H, Ph and NH), 7.19-7.30 (m, 2H, Ph), 6.77 (dd, J=9.3, 0.5 Hz, 1H, 3-H). IR: $3237(\mathrm{~N}-\mathrm{H}), 1505$ and $1335 \mathrm{~cm}^{-1}\left(\mathrm{NO}_{2}\right)$. UV, $\lambda_{\max }(\lg \varepsilon): 366$ (3.30), end absorption up to $474 \mathrm{~nm}$. MS, $m / z$ (I $\geq 10 \%): 215$ (89) [M] $]^{+}, 214$ (100), 169 (18), 168 (71) $\left[\mathrm{M}-\mathrm{HNO}_{2}\right]^{+}, 142$ (14), 115 (25), 84 (12), 78 (16), 77 (58), 65 (24), 51 (37). Anal. Calcd for $\mathrm{C}_{11} \mathrm{H}_{9} \mathrm{~N}_{3} \mathrm{O}_{2}$ : C, 61.39; $\mathrm{H}$, 4.22; N, 19.53\%. Found: C, 61.64; H, 4.30; N, 19.32\%.

$N$-(5-Nitropyridin-2-yl)benzimidamide (2d). Yellow needles, decomp. $>185{ }^{\circ} \mathrm{C}$ (heptane). ${ }^{1} \mathrm{H}$ NMR (DMSO-D, 400 MHz): $\delta 10.69$ (br s, 1H, NH), 9.21 (dd, $J=2.9,0.5$ Hz, 1H, 6'-H), 8.37 $\left(\mathrm{dd}, J=9.0,2.9 \mathrm{~Hz}, 1 \mathrm{H}, 4^{\prime}-\mathrm{H}\right), 7.96(\mathrm{~m}, 2 \mathrm{H}, \mathrm{Ph}), 7.47-7.55(\mathrm{~m}, 3 \mathrm{H}, \mathrm{Ph}), 7.30(\mathrm{~d}, J=9.0 \mathrm{~Hz}, 1 \mathrm{H}$, $\left.3^{\prime}-\mathrm{H}\right), 6.35$ (br s, 1H, NH). ${ }^{13} \mathrm{C}$ NMR (DMSO-D, $\left.100 \mathrm{MHz}\right): \delta 166.9,160.8,143.9,137.9$, 135.3, 132.3, 131.3, 128.2, 127.7, 121.1. IR: 3326 and $3162(\mathrm{~N}-\mathrm{H}), 1519$ and $1337 \mathrm{~cm}^{-1}\left(\mathrm{NO}_{2}\right)$. $\mathrm{UV}, \lambda_{\max }(\lg \varepsilon): 266$ (4.06), 314 (4.40), $376 \mathrm{sh}$ (3.57), end absorption up to $436 \mathrm{~nm}$. MS, $\mathrm{m} / z$ (I $\geq$ 10\%): 242 (36) [M] $]^{+}, 241$ (100), 195 (33) [M-HNO $]^{+}, 194$ (14), 104 (78), 77 (66), 66 (15), 51 (28). Anal. Calcd for $\mathrm{C}_{12} \mathrm{H}_{10} \mathrm{~N}_{4} \mathrm{O}_{2}$ : C, 59.50; H, 4.16; N, 23.13\%. Found: C, 59.22; H, 4.41; N, $22.86 \%$.

\section{General procedure for the synthesis of 2,4-dinitro- $N$-arylanilines (4a-m)}

Lithium arylamide was prepared by treating arylamine $(1.6 \mathrm{mmol})$ with butyl lithium $(1.7 \mathrm{mmol}$, $1.6 \mathrm{M}$ solution in hexane) in anhydrous THF $(8 \mathrm{~mL})$ at $-78 \ldots-70{ }^{\circ} \mathrm{C}$ under argon. Solution of $1,3-$ dinitrobenzene 3 (168 $\mathrm{mg}, 1 \mathrm{mmol})$ in THF ( $2 \mathrm{~mL}$ ) was added dropwise. The resulting mixture was stirred for 16-19 $\mathrm{h}$ with gradual warming to room temperature. The reaction mixture was diluted with water $(5 \mathrm{~mL})$ and extracted with ethyl acetate $(5 \times 15 \mathrm{~mL})$. The organic extract was dried over $\mathrm{MgSO}_{4}$, concentrated and purified by silica gel chromatography using the next mixtures as the eluent: dichloromethane/hexane (10:1) for 4a-d,f; dichloromethane/hexane (5:1)

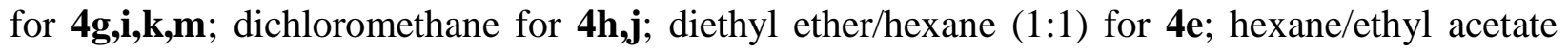
$(3: 1)$ for $4 \mathbf{l}$. From the first colorless fraction 1,3-dinitrobenzene was recovered (from the reaction with $o$-toluidine $-87 \mathrm{mg}, p$-anisidine $-47 \mathrm{mg}, o$-anisidine $-102 \mathrm{mg}, p$-nitroaniline $-72 \mathrm{mg}$, 4aminoazobenzene - $58 \mathrm{mg}$ ). The bright yellow fraction was collected. Subsequent recrystallization of the crude product from heptane gave 4 in the yield pointed in Table 2 .

$N$-(2,4-Dinitrophenyl)pyridin-2-amine (4a). Orange needles, mp 154-156 ${ }^{\circ} \mathrm{C}$ (heptane), ref. ${ }^{16}$ $155{ }^{\circ} \mathrm{C} .{ }^{1} \mathrm{H}$ NMR (DMSO-D, $250 \mathrm{MHz}$ ): $\delta 10.34$ (br s, 1H, NH), 8.79 (s, 1H, 3'-H), 8.29-8.46 (m, 2H, 3-H, 5'-H and 6-H), $7.82(\mathrm{~m}, 1 \mathrm{H}, 4-\mathrm{H}), 7.26\left(\mathrm{~d}, J=7.9 \mathrm{~Hz}, 1 \mathrm{H}, 6^{\prime}-\mathrm{H}\right), 7.19(\mathrm{~m}, 1 \mathrm{H}, 5-$ H). ${ }^{13} \mathrm{C}$ NMR (DMSO-D $\left.6,62.9 \mathrm{MHz}\right): \delta$ 153.2, 148.2, 142.7, 139.6, 139.3, 135.8, 129.9, 123.2, 121.7, 120.1, 115.8. IR: $3304(\mathrm{~N}-\mathrm{H}), 1514,1494,1335$ and $1296 \mathrm{~cm}^{-1}\left(\mathrm{NO}_{2}\right)$. UV, $\lambda_{\max }(\lg \varepsilon)$ : 355 (4.13), end absorption up to $511 \mathrm{~nm}$. MS, $m / z$ ( $\mathrm{I} \geq 10 \%): 260$ (5) $[\mathrm{M}]^{+}, 214$ (31) $\left[\mathrm{M}-\mathrm{NO}_{2}\right]^{+}$, 
168 (54) $\left[\mathrm{M}-2 \mathrm{NO}_{2}\right]^{+}, 140$ (10), 88 (12), 74 (40), 67 (12), 63 (53), 51 (100), 46 (90), 39 (63). Anal. Calcd for $\mathrm{C}_{11} \mathrm{H}_{8} \mathrm{~N}_{4} \mathrm{O}_{4}$ : C, 50.77; H, 3.10; N, 21.53\%. Found: C, 50.99; H, 3.25; N, $21.27 \%$. $\boldsymbol{N}$-(2,4-Dinitrophenyl)pyridin-4-amine (4b). Bright yellow needles, mp 198-200 ${ }^{\circ} \mathrm{C}$ (heptane). ${ }^{1} \mathrm{H}$ NMR (DMSO-D, $\left.250 \mathrm{MHz}\right): \delta 9.98$ (br s, 1H, NH), 8.84 (d, $\left.J=2.7 \mathrm{~Hz}, 1 \mathrm{H}, 3^{\prime}-\mathrm{H}\right), 8.51$ (m, 2H, 2-H and 6-H), 8.31 (dd, $\left.J=9.4,2.7 \mathrm{~Hz}, 1 \mathrm{H}, 5^{\prime}-\mathrm{H}\right), 7.59$ (d, $\left.J=9.4 \mathrm{~Hz}, 1 \mathrm{H}, 6^{\prime}-\mathrm{H}\right), 7.32$ (br d, $J=5.9 \mathrm{~Hz}, 2 \mathrm{H}, 3-\mathrm{H}$ and $5-\mathrm{H}) .{ }^{13} \mathrm{C} \mathrm{NMR}\left(\mathrm{CDCl}_{3}, 62.9 \mathrm{MHz}\right): \delta 151.7,147.0,144.1,139.5$, 135.7, 130.4, 123.9, 120.1, 117.1. IR: $3325(\mathrm{~N}-\mathrm{H}), 1514$ and $1336 \mathrm{~cm}^{-1}\left(\mathrm{NO}_{2}\right)$. UV, $\lambda_{\max }(\lg \varepsilon)$ : 325 (4.13), 351 (4.11), end absorption up to $471 \mathrm{~nm}$. MS, $m / z$ (I $\geq 10 \%): 260$ (65) [M] $]^{+}, 197$ (10), 191 (10), 184 (10), 168 (35) [M-2NO $]^{+}, 155$ (22), 140 (57), 130 (22), 114 (47), 102 (12), 93 (11), 88 (29), 78 (70), 63 (47), 51 (100), 46 (25), 39 (27). Anal. Calcd for $\mathrm{C}_{11} \mathrm{H}_{8} \mathrm{~N}_{4} \mathrm{O}_{4}$ : C, 50.77; H, 3.10; N, 21.53\%. Found: C, 51.04; H, 3.38; N, $21.42 \%$.

$N$-(2,4-Dinitrophenyl)naphthalen-2-amine (4c). Orange needles, mp 171-173 ${ }^{\circ} \mathrm{C}$ (heptane), ref. ${ }^{17} 173{ }^{\circ} \mathrm{C} .{ }^{1} \mathrm{H} \mathrm{NMR}\left(\mathrm{CDCl}_{3}, 250 \mathrm{MHz}\right): \delta 10.11$ (br s, $\left.1 \mathrm{H}, \mathrm{NH}\right), 9.14$ (d, $J=2.5 \mathrm{~Hz}, 1 \mathrm{H}, 3^{\prime}-$ H), $8.13\left(\mathrm{dd}, J=9.5,2.2 \mathrm{~Hz}, 1 \mathrm{H}, 5^{\prime}-\mathrm{H}\right), 7.96(\mathrm{~d}, J=8.5 \mathrm{~Hz}, 1 \mathrm{H}, 4-\mathrm{H}), 7.83-7.90(\mathrm{~m}, 2 \mathrm{H}$, naphthalene), $7.76(\mathrm{~d}, J=1.3 \mathrm{~Hz}, 1 \mathrm{H}, 1-\mathrm{H}), 7.51-7.58(\mathrm{~m}, 2 \mathrm{H}$, naphthalene), 7.37 (dd, $J=8.5$, $2.2 \mathrm{~Hz}, 1 \mathrm{H}, 3-\mathrm{H}), 7.21\left(\mathrm{~d}, J=9.5 \mathrm{~Hz}, 1 \mathrm{H}, 6{ }^{\prime}-\mathrm{H}\right) .{ }^{13} \mathrm{C} \mathrm{NMR}\left(\mathrm{CDCl}_{3}, 62.9 \mathrm{MHz}\right): \delta 147.4,137.9$, $134.5,134.3,132.6,131.7,130.8,130.3,128.3,128.1,127.7,127.2$, 124.5, 123.9, 123.8, 116.6. IR: $3309(\mathrm{~N}-\mathrm{H}), 1513,1347$ and $1328 \mathrm{~cm}^{-1}\left(\mathrm{NO}_{2}\right)$. UV, $\lambda_{\max }(\lg \varepsilon): 355$ (3.89), end absorption up to $521 \mathrm{~nm}$. MS, $m / z$ (I $\geq 10 \%): 309$ (100) $[\mathrm{M}]^{+}, 262$ (10), 246 (23), 217 (76) [M-2NO $]_{2}^{+}, 216$ (80), 204 (24), 189 (57), 163 (13), 152 (10), 139 (14), 127 (58), 115 (58), 109 (16), 102 (16), 94 (18), 89 (15), 77 (37), 63 (48), 51 (19), 46 (17), 44 (19), 38 (16). Anal. Calcd for $\mathrm{C}_{16} \mathrm{H}_{11} \mathrm{~N}_{3} \mathrm{O}_{4}$ : C, $62.14 ; \mathrm{H}, 3.58 ; \mathrm{N}, 13.59 \%$. Found: C, 61.79; H, 3.90; N, $13.21 \%$.

$\boldsymbol{N}$-(2,4-Dinitrophenyl)naphthalen-1-amine (4d). Orange crystals, mp 190-191 ${ }^{\circ} \mathrm{C}$ (heptane), ref. ${ }^{18} 190-191{ }^{\circ} \mathrm{C} .{ }^{1} \mathrm{H}$ NMR $\left(\mathrm{CDCl}_{3}, 250 \mathrm{MHz}\right): \delta 10.17$ (br s, $\left.1 \mathrm{H}, \mathrm{NH}\right), 9.23$ (d, J = $2.7 \mathrm{~Hz}, 1 \mathrm{H}$, 3'-H), $8.06\left(\mathrm{dd}, J=9.5,2.7 \mathrm{~Hz}, 1 \mathrm{H}, 5^{\prime}-\mathrm{H}\right), 7.92-7.99(\mathrm{~m}, 2 \mathrm{H}$, naphthalene), 7.83-7.87 (m, 1H, naphthalene), 7.47-7.62 (m, 4H, naphthalene), 6.77 (d, $\left.J=9.5 \mathrm{~Hz}, 1 \mathrm{H}, 6{ }^{\prime}-\mathrm{H}\right) .{ }^{13} \mathrm{C}$ NMR (DMSO$\left.\mathrm{D}_{6}, 62.9 \mathrm{MHz}\right): \delta 149.0,136.9,135.2,134.7,131.7,130.7,130.5,129.4,129.2,128.0,127.7$, 127.1, 126.2, 124.3, 123.5, 117.8. IR: $3313(\mathrm{~N}-\mathrm{H}), 1519$ and $1334 \mathrm{~cm}^{-1}\left(\mathrm{NO}_{2}\right)$. UV, $\lambda_{\max }(\lg \varepsilon)$ : 348 (4.12), end absorption up to $506 \mathrm{~nm}$. MS, $m / z$ ( $\mathrm{I} \geq 10 \%$ ): 309 (33) [M] $]^{+}, 229$ (10), 216 (100), 204 (25), 189 (41), 163 (14), 153(15), 139 (11), 127 (56), 115 (43), 107 (13), 101 (16), 89 (14), 77 (35), 63 (98), 52 (35), 46 (82), 39 (30). Anal. Calcd for $\mathrm{C}_{16} \mathrm{H}_{11} \mathrm{~N}_{3} \mathrm{O}_{4}$ : C, 62.14; H, 3.58; N, $13.59 \%$. Found: C, 62.50; H, 3.92; N, $13.32 \%$.

2,4-Dinitro- $N$-phenylaniline (4e). Orange-red needles, mp 157-159 ${ }^{\circ} \mathrm{C}$ (heptane), ref. ${ }^{18} 153-155$ ${ }^{\circ} \mathrm{C} .{ }^{1} \mathrm{H} \mathrm{NMR}\left(\mathrm{CDCl}_{3}, 250 \mathrm{MHz}\right): \delta 9.96$ (br s, 1H, NH), 9.15 (d, $\left.J=2.5 \mathrm{~Hz}, 1 \mathrm{H}, 3-\mathrm{H}\right), 8.15$ (dd, $J=9.5,2.5 \mathrm{~Hz}, 1 \mathrm{H}, 5-\mathrm{H}), 7.46-7.53(\mathrm{~m}, 2 \mathrm{H}, \mathrm{Ph}), 7.24-7.40(\mathrm{~m}, 3 \mathrm{H}, \mathrm{Ph}), 7.15(\mathrm{~d}, J=9.5 \mathrm{~Hz}, 1 \mathrm{H}$, 6-H). ${ }^{13} \mathrm{C} \mathrm{NMR}\left(\mathrm{CDCl}_{3}, 62.9 \mathrm{MHz}\right): \delta 147.5,137.8,137.1,131.5,130.7,130.3,128.2,125.9$, 124.5, 116.5. IR: $3319(\mathrm{~N}-\mathrm{H}), 1518,1495$ and $1336 \mathrm{~cm}^{-1}\left(\mathrm{NO}_{2}\right)$. UV, $\lambda_{\max }(\lg \varepsilon): 355$ (4.07), end absorption up to $530 \mathrm{~nm}$. MS, $\mathrm{m} / z$ (I $\geq 10 \%$ ): 259 (100) [M] $]^{+}, 196$ (16), 182 (12), 167 (58) [M2NO $]^{+}, 154$ (19), 139 (41), 128 (11), 113 (10), 97 (13), 89 (12), 83 (16), 77 (61), 69 (28), 63 
(34), 57 (52), 51 (64), 46 (49), 43 (89), 38 (14). Anal. Calcd for $\mathrm{C}_{12} \mathrm{H}_{9} \mathrm{~N}_{3} \mathrm{O}_{4}$ : C, 55.60; H, 3.50; N, 16.21\%. Found: C, 55.84; H, 3.17; N, 15.98\%.

2,4-Dinitro- $N$-p-tolylaniline (4f). Orange needles, mp $133-135{ }^{\circ} \mathrm{C}$ (heptane), ref. ${ }^{18} 134-135{ }^{\circ} \mathrm{C}$. ${ }^{1} \mathrm{H}$ NMR $\left(\mathrm{CDCl}_{3}, 250 \mathrm{MHz}\right): \delta 9.91$ (br s, $\left.1 \mathrm{H}, \mathrm{NH}\right), 9.14$ (d, $\left.J=2.5 \mathrm{~Hz}, 1 \mathrm{H}, 3-\mathrm{H}\right), 8.12(\mathrm{dd}, J=$ 9.5, $2.5 \mathrm{~Hz}, 1 \mathrm{H}, 5-\mathrm{H}), 7.07-7.30\left(\mathrm{~m}, 5 \mathrm{H}, p-\mathrm{Tol}\right.$ and 6-H), $2.40(\mathrm{~s}, 3 \mathrm{H}, \mathrm{Me}) .{ }^{13} \mathrm{C} \mathrm{NMR}\left(\mathrm{CDCl}_{3}\right.$, 62.9 MHz): $\delta 147.9,138.3,137.6,134.4,131.3,131.2,130.3,126.0,124.5,116.5,21.5$. IR: 3311 $(\mathrm{N}-\mathrm{H}), 1519,1337$ and $1318 \mathrm{~cm}^{-1}\left(\mathrm{NO}_{2}\right)$. UV, $\lambda_{\max }(\lg \varepsilon): 355$ (4.19), end absorption up to 513 nm. MS, $m / z$ (I $\geq 10 \%): 273$ (100) $[\mathrm{M}]^{+}, 226$ (11), 210 (11), 180 (93) [M-2NO $\left.2-\mathrm{H}\right]^{+}, 152$ (17), 139 (13), 91 (18), 77 (16), 65 (20), 51 (11), 46 (16), 39 (14). Anal. Calcd for $\mathrm{C}_{13} \mathrm{H}_{11} \mathrm{~N}_{3} \mathrm{O}_{4}$ : C, $57.14 ; \mathrm{H}, 4.06 ; \mathrm{N}, 15.38 \%$. Found: C, 56.86; H, 4.23; N, $15.65 \%$.

2,4-Dinitro- $\boldsymbol{N}$-o-tolylaniline (4g). Orange needles, mp 124-126 ${ }^{\circ} \mathrm{C}$ (heptane), ref. ${ }^{18} 125-126{ }^{\circ} \mathrm{C}$. ${ }^{1} \mathrm{H} \mathrm{NMR}\left(\mathrm{CDCl}_{3}, 250 \mathrm{MHz}\right): \delta 9.81$ (br s, $\left.1 \mathrm{H}, \mathrm{NH}\right), 9.19$ (d, $\left.J=2.7 \mathrm{~Hz}, 1 \mathrm{H}, 3-\mathrm{H}\right), 8.13(\mathrm{dd}, J=$ 9.5, 2.7 Hz, 1H, 5-H), 7.23-7.40 (m, 4H, o-Tol), 6.81 (d, J = 9.5 Hz, 1H, 6-H), 2.25 (s, 3H, Me). ${ }^{13} \mathrm{C}$ NMR (DMSO-D, $\left.62.9 \mathrm{MHz}\right): \delta 148.1,137.0,136.7,136.1,132.2,131.2,130.8,128.9$, 128.4, 128.2, 124.3, 117.2, 18.2. IR: $3330(\mathrm{~N}-\mathrm{H}), 1515,1497$ and $1340 \mathrm{~cm}^{-1}\left(\mathrm{NO}_{2}\right) . \mathrm{UV}, \lambda_{\max }(\mathrm{lg}$ $\varepsilon): 348$ (4.20), end absorption up to $500 \mathrm{~nm}$. MS, $m / z$ ( $\mathrm{I} \geq 10 \%): 273$ (37) [M] $]^{+}, 180$ (100) [M2NO $2-\mathrm{H}]^{+}, 152$ (39), 139 (20), 128 (15), 115 (10), 91 (38), 77 (48), 65 (74), 51 (38), 46 (92), 39 (60). Anal. Calcd for $\mathrm{C}_{13} \mathrm{H}_{11} \mathrm{~N}_{3} \mathrm{O}_{4}$ : C, 57.14; H, 4.06; N, 15.38\%. Found: C, 57.41; H, 4.33; N, $15.12 \%$.

$N$-(4-Methoxyphenyl)-2,4-dinitroaniline (4h). Orange-red needles, mp $139-141{ }^{\circ} \mathrm{C}$ (heptane), ref. ${ }^{18} 139-140{ }^{\circ} \mathrm{C} .{ }^{1} \mathrm{H} \mathrm{NMR}\left(\mathrm{CDCl}_{3}, 250 \mathrm{MHz}\right): \delta 9.85$ (br s, $\left.1 \mathrm{H}, \mathrm{NH}\right), 9.16(\mathrm{~d}, J=2.6 \mathrm{~Hz}, 1 \mathrm{H}, 3-$ H), $8.12(\mathrm{dd}, J=9.6,2.6 \mathrm{~Hz}, 1 \mathrm{H}, 5-\mathrm{H}), 7.20$ (d, $J=9.0 \mathrm{~Hz}, 2 \mathrm{H}, 3^{\prime}-\mathrm{H}$ and $\left.5^{\prime}-\mathrm{H}\right), 6.99$ (m, 3H, 6$\mathrm{H}, 2^{\prime}-\mathrm{H}$ and $\left.6^{\prime}-\mathrm{H}\right), 3.85$ (s, 3H, OMe). ${ }^{13} \mathrm{C}$ NMR (DMSO-D, $\left.62.9 \mathrm{MHz}\right): \delta 159.1,148.4,136.8$, 131.4, 131.0, 130.6, 128.6, 124.3, 117.6, 115.8, 56.3. IR: 3314-3341 (N-H), 1520, 1511, 1335 and $1320 \mathrm{~cm}^{-1}\left(\mathrm{NO}_{2}\right) . \mathrm{UV}, \lambda_{\max }(\lg \varepsilon): 274$ (3.75), 356 (4.09), end absorption up to $526 \mathrm{~nm}$. MS, $m / z(\mathrm{I} \geq 10 \%): 289$ (100) [M] $]^{+}, 274$ (21), 242 (14), 227 (14), 209 (17), 197 (11) [M-2NO $\mathrm{NO}_{2}^{+}, 196$ (11), 182 (54), 154 (64), 126 (34), 123 (37), 92 (14), 77 (29), 63 (43), 51 (21), 46 (22). Anal. Calcd for $\mathrm{C}_{13} \mathrm{H}_{11} \mathrm{~N}_{3} \mathrm{O}_{5}$ : C, 53.98; H, 3.83; N, 14.53\%. Found: C, 54.29; H, 3.63; N, $14.25 \%$.

$N$-(2-Methoxyphenyl)-2,4-dinitroaniline (4i). Orange-red needles, mp 165-166 ${ }^{\circ} \mathrm{C}$ (heptane), ref. ${ }^{19} 167-168{ }^{\circ} \mathrm{C}$ (ethanol). ${ }^{1} \mathrm{H} \mathrm{NMR}\left(\mathrm{CDCl}_{3}, 250 \mathrm{MHz}\right): \delta 9.86$ (br s, $\left.1 \mathrm{H}, \mathrm{NH}\right), 9.16$ (d, $J=2.5$ $\mathrm{Hz}, 1 \mathrm{H}, 3-\mathrm{H}), 8.15(\mathrm{dd}, J=9.6,2.5 \mathrm{~Hz}, 1 \mathrm{H}, 5-\mathrm{H}), 7.26-7.38\left(\mathrm{~m}, 2 \mathrm{H}, 3^{\prime}-\mathrm{H}\right.$ and $\left.6^{\prime}-\mathrm{H}\right), 7.09(\mathrm{~d}, J=$ $9.6 \mathrm{~Hz}, 1 \mathrm{H}, 6-\mathrm{H}), 6.98-7.07\left(\mathrm{~m}, 2 \mathrm{H}, 4^{\prime}-\mathrm{H}\right.$ and $\left.5^{\prime}-\mathrm{H}\right), 3.85(\mathrm{~s}, 3 \mathrm{H}, \mathrm{OMe}) .{ }^{13} \mathrm{C} \mathrm{NMR}\left(\mathrm{CDCl}_{3}, 62.9\right.$ MHz): $\delta 153.9,147.2,137.7,131.8,130.0,128.9,125.9,125.8,124.4,121.5,116.7,112.5,56.2$. IR: $3312(\mathrm{~N}-\mathrm{H}), 1521,1499,1337$ and $1303 \mathrm{~cm}^{-1}\left(\mathrm{NO}_{2}\right)$. UV, $\lambda_{\max }(\lg \varepsilon): 262$ (3.58), 272 (3.81), 362 (4.11), end absorption up to $531 \mathrm{~nm}$. MS, $m / z$ (I $\geq 10 \%): 289$ (100) [M] ${ }^{+}, 257$ (16), 227 (14), 182 (16), 154 (17), 127 (12), 77 (26), 63 (32), 51 (24), 39 (17). Anal. Calcd for $\mathrm{C}_{13} \mathrm{H}_{11} \mathrm{~N}_{3} \mathrm{O}_{5}$ : C, $53.98 ; \mathrm{H}, 3.83$; N, 14.53\%. Found: C, 53.72; H, 3.50; N, $14.81 \%$.

2,4-Dinitro- $\boldsymbol{N}$-(4-nitrophenyl)aniline (4j). Yellow needles, mp 185-187 ${ }^{\circ} \mathrm{C}$ (heptane), ref. $^{20}$ 186-186.7 ${ }^{\circ} \mathrm{C} .{ }^{1} \mathrm{H} \mathrm{NMR}\left(\mathrm{CDCl}_{3}, 250 \mathrm{MHz}\right): \delta 10.04$ (br s, $\left.1 \mathrm{H}, \mathrm{NH}\right), 9.19$ (d, J = 2.5 Hz, 1H, 3$\mathrm{H})$, 8.27-8.38 (m, 3H, 3'-H, 5'-H and 5-H), 7.41-7.49 (m, 3H, 2'-H, 6'-H and 6-H). ${ }^{13} \mathrm{C}$ NMR 
(DMSO-D 6 , 62.9 MHz): $\delta$ 146.2, 144.4, 139.6, 135.7, 130.4, 126.2, 123.9, 123.4, 123.3, 120.3. IR: $3308(\mathrm{~N}-\mathrm{H}), 1515$ and $1334 \mathrm{~cm}^{-1}\left(\mathrm{NO}_{2}\right) . \mathrm{UV}, \lambda_{\max }(\lg \varepsilon): 274$ (4.17), 360 (4.36), end absorption up to $490 \mathrm{~nm}$. MS, $m / z$ (I $\geq 5 \%$ ): 304 (100) [M] $]^{+}, 274$ (12), 241 (5), 182 (9), 166 (16), 154 (9), 139 (20), 76 (5), 63 (11). Anal. Calcd for $\mathrm{C}_{12} \mathrm{H}_{8} \mathrm{~N}_{4} \mathrm{O}_{6}$ : C, 47.38; H, 2.65; N, 18.42\%. Found: C, 47.09; H, 2.54; N, 18.23\%.

2,4-Dinitro- $N$-(4-(phenyldiazenyl)phenyl)aniline (4k). Red needles, mp 174-176 ${ }^{\circ} \mathrm{C}$ (heptane), ref. ${ }^{21} .{ }^{1} \mathrm{H} \mathrm{NMR}\left(\mathrm{CDCl}_{3}, 250 \mathrm{MHz}\right): \delta 10.06$ (br s, $\left.1 \mathrm{H}, \mathrm{NH}\right), 9.19(\mathrm{~d}, J=2.2 \mathrm{~Hz}, 1 \mathrm{H}, 3-\mathrm{H}), 8.23$ $(\mathrm{dd}, J=9.5,2.2 \mathrm{~Hz}, 1 \mathrm{H}, 5-\mathrm{H}), 8.04\left(\mathrm{~d}, J=8.5 \mathrm{~Hz}, 2 \mathrm{H}, 3^{\prime}-\mathrm{H}\right.$ and $\left.5^{\prime}-\mathrm{H}\right), 7.93(\mathrm{~m}, 2 \mathrm{H}, \mathrm{Ph}), 7.52$ $(\mathrm{m}, 3 \mathrm{H}, \mathrm{Ph}), 7.45$ (d, $J=8.5 \mathrm{~Hz}, 2 \mathrm{H}, 2^{\prime}-\mathrm{H}$ and $\left.6^{\prime}-\mathrm{H}\right), 7.35$ (d, $\left.J=9.5 \mathrm{~Hz}, 1 \mathrm{H}, 6-\mathrm{H}\right) .{ }^{13} \mathrm{C} \mathrm{NMR}$ $\left(\mathrm{CDCl}_{3}, 62.9 \mathrm{MHz}\right): \delta 152.9,151.5,146.5,139.4,138.4,132.3,131.9,130.5,129.6,125.5$, 125.1, 124.5, 123.4, 116.7. IR: $3299(\mathrm{~N}-\mathrm{H}), 1514$ and $1340 \mathrm{~cm}^{-1}\left(\mathrm{NO}_{2}\right)$. UV, $\lambda_{\max }(\lg \varepsilon): 368$ (4.26), end absorption up to $552 \mathrm{~nm}$. MS, $m / z$ (I $\geq 10 \%): 363$ (16) [M] $]^{+}, 258$ (26), 139 (14), 105 (13), 92 (18), 77 (98), 63 (21), 51 (47), 39 (12). Anal. Calcd for $\mathrm{C}_{18} \mathrm{H}_{13} \mathrm{~N}_{5} \mathrm{O}_{4}$ : C, 59.50; H, 3.61; N, 19.28\%. Found: C, 59.58; H, 3.44; N, $19.56 \%$.

$\boldsymbol{N}$-(2,4-Dinitrophenyl)thiazol-2-amine (4l). Orange crystals, mp 153-155 ${ }^{\circ} \mathrm{C}$ (heptane). ${ }^{1} \mathrm{H}$ NMR (DMSO-D, $250 \mathrm{MHz}): \delta 11.0$ (br s, 1H, NH), 8.80 (d, $\left.J=2.8 \mathrm{~Hz}, 1 \mathrm{H}, 3^{\prime}-\mathrm{H}\right), 8.59$ (d, $J=$ $\left.9.5 \mathrm{~Hz}, 1 \mathrm{H}, 6^{\prime}-\mathrm{H}\right), 8.45\left(\mathrm{dd}, J=9.5,2.5 \mathrm{~Hz}, 1 \mathrm{H}, 5^{\prime}-\mathrm{H}\right), 7.50(\mathrm{~d}, J=3.8 \mathrm{~Hz}, 1 \mathrm{H}, 5-\mathrm{H}), 7.36(\mathrm{~d}, J=$ $3.8 \mathrm{~Hz}, 1 \mathrm{H}, 4-\mathrm{H}) .{ }^{13} \mathrm{C}$ NMR (DMSO-D $\left.6,62.9 \mathrm{MHz}\right): \delta 161.9,142.1,139.8,135.6(1), 135.6(0)$, 130.3, 123.0, 120.8, 115.9. IR: $3293(\mathrm{~N}-\mathrm{H}), 1519,1504,1338$ and $1311 \mathrm{~cm}^{-1}\left(\mathrm{NO}_{2}\right) . \mathrm{UV}, \lambda_{\max }(\mathrm{lg}$ $\varepsilon)$ : 266 (4.06), 356 (4.20), end absorption up to $518 \mathrm{~nm}$. MS, $m / z$ (I $\geq 10 \%): 266$ (100) [M] $]^{+}, 249$ (31), 220 (18) [M-NO $]^{+}, 193$ (50), 174 (31), [M-2NO $]^{+}, 129$ (11), 103 (14), 100 (24), 75 (33), 63 (43), 58 (84), 50 (19), 45 (72), 40 (20). Anal. Calcd for $\mathrm{C}_{9} \mathrm{H}_{6} \mathrm{~N}_{4} \mathrm{O}_{4} \mathrm{~S}: \mathrm{C}, 40.60 ; \mathrm{H}, 2.27 ; \mathrm{N}$, 21.04 ; S, 12.04\%. Found: C, 40.25; H, 2.03; N, 21.36; S, $11.70 \%$.

$\boldsymbol{N}$-(2,4-Dinitrophenyl)benzamide (4m). Yellow needles, mp 194-196 ${ }^{\circ} \mathrm{C}$ (heptane). ${ }^{1} \mathrm{H}$ NMR (DMSO-D, $250 \mathrm{MHz}$ ): $\delta 11.23$ (br s, 1H, NH), 8.76 (d, $\left.J=2.3 \mathrm{~Hz}, 1 \mathrm{H}, 3{ }^{\prime}-\mathrm{H}\right), 8.60$ (dd, $J=9.1$, $\left.2.3 \mathrm{~Hz}, 1 \mathrm{H}, 5^{\prime}-\mathrm{H}\right), 8.14\left(\mathrm{~d}, J=9.1 \mathrm{~Hz}, 1 \mathrm{H}, 6^{\prime}-\mathrm{H}\right), 7.99$ (d, $J=7.5 \mathrm{~Hz}, 2 \mathrm{H}, 2-\mathrm{H}$ and 6-H), 7.59$7.73\left(\mathrm{~m}, 3 \mathrm{H}, 3-\mathrm{H}, 4-\mathrm{H}\right.$ and 5-H). ${ }^{13} \mathrm{C}$ NMR (DMSO-D, $\left.62.9 \mathrm{MHz}\right): \delta 166.4,143.7,141.8,138.2$, 133.8, 133.7, 129.7, 129.5, 128.8, 126.4, 122.1. IR: 3102-3132 (N-H), $1699(\mathrm{C}=\mathrm{O}), 1339 \mathrm{~cm}^{-1}$ $\left(\mathrm{NO}_{2}\right)$. UV, $\lambda_{\max }(\lg \varepsilon): 304$ (4.57), end absorption up to $434 \mathrm{~nm}$. MS, $m / z$ (I $\left.\geq 5 \%\right): 287$ (7) [M] $]^{+}$, 105 (100), 77 (50), 51 (14). Anal. Calcd for $\mathrm{C}_{13} \mathrm{H}_{9} \mathrm{~N}_{3} \mathrm{O}_{5}$ : C, 54.36; H, 3.16; N, 14.63\%. Found: C, $54.08 ; \mathrm{H}, 3.33 ; \mathrm{N}, 14.39 \%$.

\section{Acknowledgements}

The authors would like to thank Zoya A. Starikova (A. N. Nesmeyanov Institute of Organoelement Compounds, Russian Academy of Sciences) for the X-Ray analysis. 


\section{References}

1. (a) Corey, E. J.; Czakó, B.; Kürti, L. Molecules and Medicine; Wiley: Hoboken, New Jersey, 2007. (b) Travis, A. S. In The Chemistry of Anilines; Patai Series The Chemistry of Functional Groups, Ed. Rappoport, Z.; Wiley: Chichester, 2007, Part 2, Chapter 13, pp 715782. (c) Ning, Z.; Tian, H. Chem. Commun., 2009, 5483.

2. (a) Gorelik, M. V.; Efros, L. S. Osnovy khimii i tekhnologii aromaticheskih soedineniy; Khimia: Moscow, 1992; pp 302-340. (b) Terrier, F. Nucleophilic Aromatic Displacement: The Influence of the Nitro Group; In Organic Nitro Chemistry Series, VCH Publishers, Inc.: New York, 1991.

3. (a) Wolfe, J. P.; Tomori, H.; Sadighi, J. P.; Yin, J.; Buchwald, S. L. J. Org. Chem. 2000, 65, 1158. (b) Surry, D. S.; Buchwald, S. L. Angew. Chem. Int. Ed. 2008, 47, 6338.

4. (a) Chupakhin, O. N.; Charushin, V. N.; van der Plas, H.C. Nucleophilic Aromatic Substitution of Hydrogen; Academic Press: San Diego, 1994, p 367. (b) Mąkosza, M.; Wojciechowski, K. Chem. Rev. 2004, 104, 2631.

5. (a) van der Plas, H. C. Adv. Heterocycl. Chem. 2004, 86, 1. (b) Gulevskaya, A. V.; Pozharskii, A. F. Izv. Akad. Nauk, Ser. Khim. 2008, 899 (Russ. Chem. Bull. 2008, 57, 913). (c) Gulevskaya, A. V.; Maes, B. U. W.; Meyers, C.; Herrebout, W. A.; Van der Veken, B. J. Eur. J. Org. Chem. 2006, 5305. (d) Gulevskaya, A. V.; Burov, O. N.; Pozharskii, A. F.; Kletskii, M. E.; Korbukova, I. N. Tetrahedron 2008, 64, 696. (e) Gulevskaya, A. V.; Verbeeck, S.; Burov, O. N.; Meyers, C.; Korbukova, I. N.; Herrebout, W.; Maes, B. U. W. Eur. J. Org. Chem. 2009, 564. (f) Burov, O. N.; Gulevskaya, A. V.; Pozharskii, A. F. Khim. Geterotsikl. Soedin. 2009, 602 (Chem. Heterocycl. Comp. 2009, 45, 475).

6. (a) Mąkosza, M. Izv. Akad. Nauk, Ser. Khim. 1996, 531 (Russ. Chem. Bull. 1996, 45, 491).

(b) Bakke, J. M.; Svensen, H.; Trevisan, R. J. Chem. Soc. Perkin Trans. 1, 2001, 376. (c) Mąkosza, M.; Białecki, M. J. Org. Chem. 1998, 63, 4878. (d) Rozhkov, V. V.; Shevelev, S. A.; Chervin, I. I.; Mitchell, A. R.; Schmidt, R. D. J. Org. Chem. 2003, 68, 2498. (e) Grzegozek, M. J. Heterocycl. Chem. 2008, 45, 1879. (f) Szpakiewicz, B.; Grzegozek, M. Can. J. Chem. 2008, 86, 682.

7. For review see: Gulevskaya, A. V.; Pozharskii, A. F. Adv. Heterocycl. Chem. 2007, 93, 57.

8. (a) Grabenwöger, M.; Haider, N.; Heinish, G. Liebigs Ann. Chem. 1989, 481. (b) Boguslavskiy, A. M.; Ponizovskiy, M. G.; Kodess, M. I.; Charushin, V. N. Izv. Akad. Nauk, Ser. Khim. 2003, 2060 (Russ. Chem. Bull. 2003, 52, 2175).

9. Esser, F.; Pook, K.-H. Synthesis 1992, 596.

10. Budyka, M. F.; Terent'ev, P. B.; Kost, A. N. Khim. Geterotsikl. Soedin. 1978, 809 (Chem. Heterocycl. Comp., 1978, 14, 663).

11. Garnier, E.; Audoux, J.; Pasquinet, E.; Suzenet, F.; Poullain, D.; Lebret, B.; Guillaumet, G. J. Org. Chem. 2004, 69, 7809.

12. Patriciu, O.-I.; Fînaru, A.-L.; Săndulescu, I.; Guillaumet, G. Synthesis 2007, 3868.

13. Bordwell pKa Table (Acidity in DMSO) (http://www.chem.wisc.edu/areas/reich/pkatable/). 
14. Tschitschibabin, A. E.; Preobrashensky, W. A. Ber. 1928, 61, 199.

15. Bell, M. G. W.; Day, M.; Peters, A. T. J. Chem. Soc. C 1967, 132.

16. Sharnin, G. P.; Falyakhov, I. F.; Khairutdinov, F. G. Khim. Geterotsikl. Soedin. 1983, 635 (Chem. Heterocycl. Comp., 1983, 19, 511).

17. Vorozhtsov, N.N.; Jakobson, G. G. Zh. Obsh. Khim. 1958, 28, 40.

18. Vizgert, R. V., Berkman, J. P. Zh. Obsh. Khim. 1958, 28, 2119.

19. Nudelman, N. S.; Palleros, D. J. Org. Chem. 1983, 48, 1613.

20. Davis, T. L.; Ashdown, A. A. J. Am. Chem. Soc. 1924, 46, 1051.

21. von Walther, R.; Lehmann, A. J. Prakt. Chem. 1904, 69, 42. 